\title{
Interference Management for DS-CDMA Systems through Closed-Loop Power Control, Base Station Assignment, and Beamforming
}

\author{
Mohamad Dosaranian Moghadam ${ }^{1}$, Hamidreza Bakhshi², Gholamreza Dadashzadeh ${ }^{2}$ \\ ${ }^{1}$ Department of Electrical Engineering, Science and Research Branch of Islamic Azad University, Tehran, Iran \\ ${ }^{2}$ Department of Electrical Engineering, Shahed University, Tehran, Iran \\ E-mail:m_dmoghadam@qiau.ac.ir, \{bakhshi,gdadashzadeh\}@shahed.ac.ir \\ Received April 8, 2010; revised May 15, 2010; accepted May 23, 2010
}

\begin{abstract}
In this paper, we propose a smart step closed-loop power control (SSPC) algorithm and a base station assignment method based on minimizing the transmitter power (BSA-MTP) technique in a direct sequence-code division multiple access (DS-CDMA) receiver with frequency-selective Rayleigh fading. This receiver consists of three stages. In the first stage, with constrained least mean squared (CLMS) algorithm, the desired users' signal in an arbitrary path is passed and the inter-path interference (IPI) is reduced in other paths in each RAKE finger. Also in this stage, the multiple access interference (MAI) from other users is reduced. Thus, the matched filter (MF) can use for more reduction of the IPI and MAI in each RAKE finger in the second stage. Also in the third stage, the output signals from the matched filters are combined according to the conventional maximal ratio combining (MRC) principle and then are fed into the decision circuit of the desired user. The simulation results indicate that the SSPC algorithm and the BSA-MTP technique can significantly reduce the network bit error rate (BER) compared to the other methods. Also, we observe that significant savings in total transmit power (TTP) are possible with our methods.
\end{abstract}

Keywords: Adaptive Beamforming, Antenna Array, Base Station Assignment, Closed-Loop Power Control, Constrained LMS, DS-CDMA

\section{Introduction}

Code-Division Multiple Access (CDMA) for cellular communication networks requires the implementation of some forms of adaptive power control. In uplink of CDMA systems, the maximum number of supportable users per cell is limited by multipath fading, shadowing, and near-far effects that cause fluctuations of the received power at the base station (BS). Two types of power control are often considered: closed-loop power control and open-loop power control $[1,2]$. In a closed-loop power control, according to the received signal power at a base station, the base station sends a command to a mobile set to adjust the transmit power of the mobile. Also, closedloop power control is employed to combat fast channel fluctuations due to fading. Closed-loop algorithms can effectively compensate fading variations when the power control updating time is smaller than the correlation time of the channel. However, in an open-loop power control, a mobile user adjusts its transmit power according to its received power in downlink [1-5]. In this paper, an adaptive closed-loop power control algorithm is proposed to compensate for near-far effects.

Diversity and power control are two effective techniques for enhancing the signal-to-interference-plus-noise ratio (SINR) for wireless networks. Diversity exploits the random nature of radio propagation by finding independent (or, at least, highly uncorrelated) signal paths for communication. If one radio path undergoes a deep fade, another independent path may have a strong signal. By having more than one path to select from, the SINR at the receiver can be improved. The diversity scheme can be divided into three methods: 1) The space diversity; 2) The time diversity; 3) The frequency diversity. In these schemes, the same information is first received (or transmitted) at different locations (or time slots/frequency bands). After that, these signals are combined to increase the received SINR. The antenna array is an example of the space diversity, which uses a beamformer to increase the SINR for a particular direction [6-8]. 
The first goal of this paper is to extend the works in [9] and [10] by considering multiple-cell system and closedloop power control. In these works, a RAKE receiver in single-cell system with conjugate gradient adaptive beamforming was proposed in the presence of frequencyselective Rayleigh fading channel, and perfect power control (PPC) was considered.

In this work, the performance analysis of direct sequence (DS)-CDMA system in frequency-selective Rayleigh fading channel has been studied. If the delay spread in a multipath channel is larger than a fraction of a symbol, the delayed components will cause inter-symbol interference (ISI). Adaptive receiver beamforming schemes have been widely used to reduce both co-channel interference (CCI) and ISI and to decrease the bit error rate (BER) by adjusting the beam pattern such that the effective SINR at the output of the beamformer is optimally increased [11].

In this paper a RAKE receiver in DS-CDMA system is analyzed in three stages according to Figure 1 [9]. In the first stage, this receiver uses constrained least mean squared (CLMS) adaptive beamforming algorithm to find optimum antenna weights assuming perfect estimation of the channel parameters (direction, delay, and power) for the desired user. The desired user resolvable paths' directions are fed to the beamformer to reduce the inter-path interference (IPI) from other directions. Also, the RAKE receiver uses conventional demodulation in the second stage and conventional maximal ratio combining (MRC) in the third stage to reduce multiple access interference (MAI) and the other interferences. Reducing the MAI and CCI will further decrease the system BER.

To improve the performance of cellular systems, base station assignment (BSA) technique can be used. In the joint power control and base station assignment, a number of base stations are potential receivers of a mobile transmitter. Here, the objective is to determine the assignment of users to base stations which minimizes the allocated mobile powers [12-15]. In simple mode and in multiple-cell systems, the user is connected to the nearest base station. This way is not optimal in cellular systems under the shadowing and multipath fading channels and can increase the system BER.

Accordingly, the second goal of this paper is to use base station assignment technique. In [14], the combined the base station assignment and power control was used to increase uplink capacity in cellular communication networks. In that work, it was shown that if there exists at least one feasible base station assignment, the proposed algorithm will find the jointly optimal base station assignment and minimal transmitter power level for all users. In this paper, we present the base station assignment method based on minimizing the transmitter power (BSA-MTP) for decreasing the BER in all cells.

The organization of the remainder of this paper is as follows. The system model is presented in Section 2. The RAKE receiver structure is described in Section 3. In Section 4, we propose smart step closed-loop power control (SSPC) algorithm. In Section 5, the BSA-MTP technique is presented. Section 6 describes switched-beam (SB) technique and equal sectoring (ES) method. Finally, simulation results and conclusions are given in Section 7 and Section 8 , respectively.

\section{System Model}

In this paper, we focus on the uplink communication paths in a DS-CDMA cellular system. $L$ replicas of the signal, due to both some form of diversity reception (for instance antenna diversity) and channel frequency selectivity, are assumed Rayleigh distributed and optimally combined through a RAKE receiver according to Figure 1.

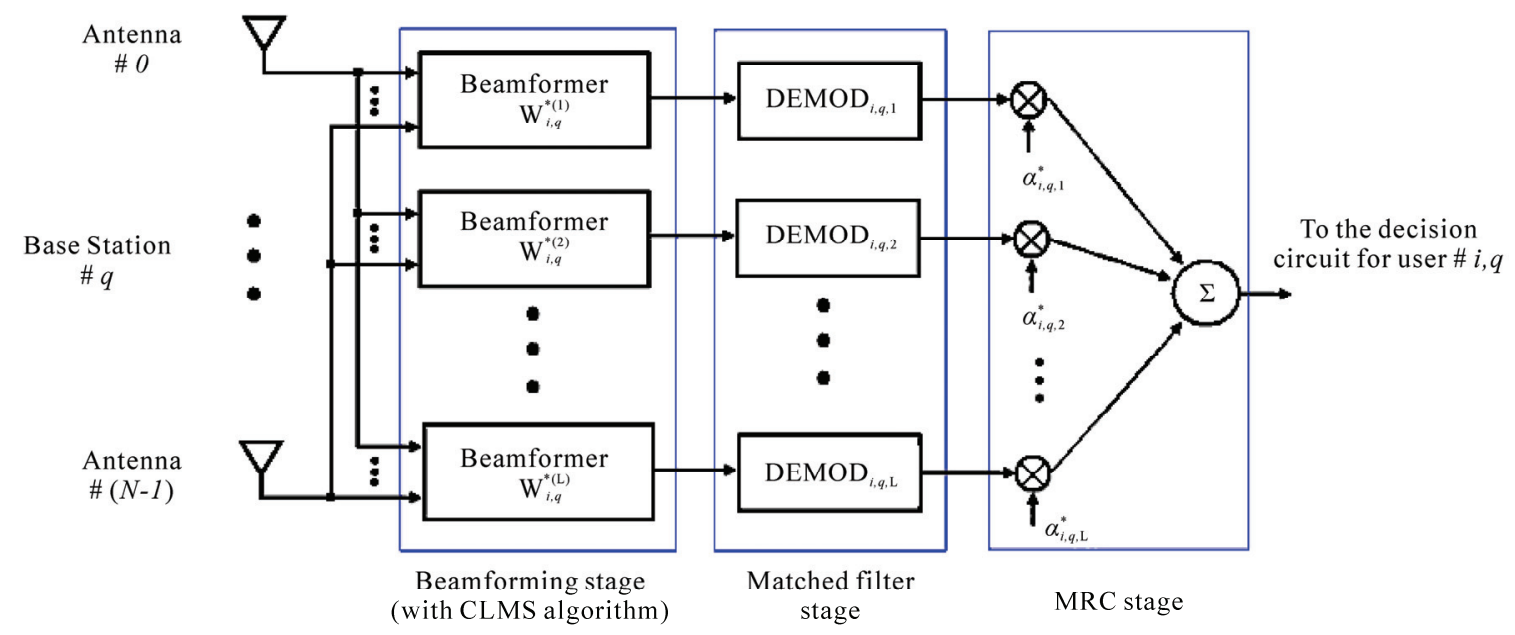

Figure 1. Block diagram of a three-stage RAKE receiver in DS-CDMA system [9]. 
Also assume that there are $M$ active base stations in the network, with $K_{m}$ users connected to $m$ th base station, where $1 \leq m \leq M$. Also assume that each base station uses an antenna array of $S$ sensors and $N$ weights, where $S=N$, to receive signals from all users. Also, for simplicity we assume a synchronous DSCDMA scheme and BPSK modulation in order to simplify the analysis of proposed methods. Additionally, in this paper we assume a slow fading channel. Hence, the received signal in the base station $q$ and sensor $s$ from all users can be written as $[9,16]$

$$
\begin{array}{r}
r_{q, s}(t)=\sum_{k} \sqrt{p_{k, m}^{\prime} \Gamma_{k}(x, y)} \sum_{l=1}^{L} \alpha_{k, m, l} b_{k, m}\left(t-\tau_{k, m, l}\right) \\
\times c_{k, m}\left(t-\tau_{k, m, l}\right) \exp \left(-j s k_{d} \sin \theta_{k, m, l}\right)+n(t)
\end{array}
$$

where $c_{k, m}(t)$ is the pseudo noise (PN) chips of user $k$ in cell $m$ (user $k, m$ ) with a chip period of $T_{c}$; $b_{k, m}(t)$ is the information bit sequence of user $k, m$ with a bit period of $T_{b}=G T_{c}$ where $G$ is processing gain; $\tau_{k, m, l}$ is the $l$ th path time delay for user $k, m$; $\theta_{k, m, l}$ is the direction of arrival (DoA) in the $l$ th path for user $k, m ; \alpha_{k, m, l}$ is the complex Gaussian fading channel coefficient from the lth path of user $k, m$; $k_{d}=2 \pi d / \lambda$ where $\lambda$ is signal wavelength and $d$ is the distance between the antenna elements that for avoid the spatial aliasing should be defined as $d=0.5 \lambda$ and $n(t)$ is an additive white Gaussian noise (AWGN) process with a two-sided power spectral density (PSD) of $N_{0} / 2$. Also for conventional BSA technique, $\Gamma_{k}(x, y)$ is defined as

$$
\Gamma_{k}(x, y)=\left\{\begin{array}{cl}
1 & ; k \in S_{\mathrm{BS} q} \\
\frac{\min _{m \in \Theta_{k}}\left\{d_{k, m}^{L_{\alpha}}(x, y) 10^{\xi_{k, m} / 10}\right\}}{d_{k, q}^{L_{\alpha}}(x, y) 10^{\xi_{k, q} / 10}} & ; k \in S_{o}
\end{array}\right.
$$

where $L_{\alpha}$ is path-loss exponent; $d_{k, m}(x, y)$ and $d_{k, q}(x, y)$ are the distance between user $k$ and $\operatorname{BS} m$ and $\operatorname{BS} q$, respectively (see Figure 2). Also the variable $\Theta_{k}$ defined the set of the nearest BSs to user $k ; \xi_{k, m}$ is a random variable modeling the shadowing between user $k$ and $\mathrm{BS} m ; S_{\mathrm{BS} q}$ is the set of users that connected to $\operatorname{BS} q$ and $S_{o}$ is the set of users that not connected to $\mathrm{BS} q$ [2]. Also in (1)

$$
p_{k, m}^{\prime}=d_{k, m}^{-L_{\alpha}}(x, y) 10^{-\xi_{k, m} / 10} \times p_{k, m}
$$

is the received power in the $\mathrm{BS} m$ of user $k, m$ in the

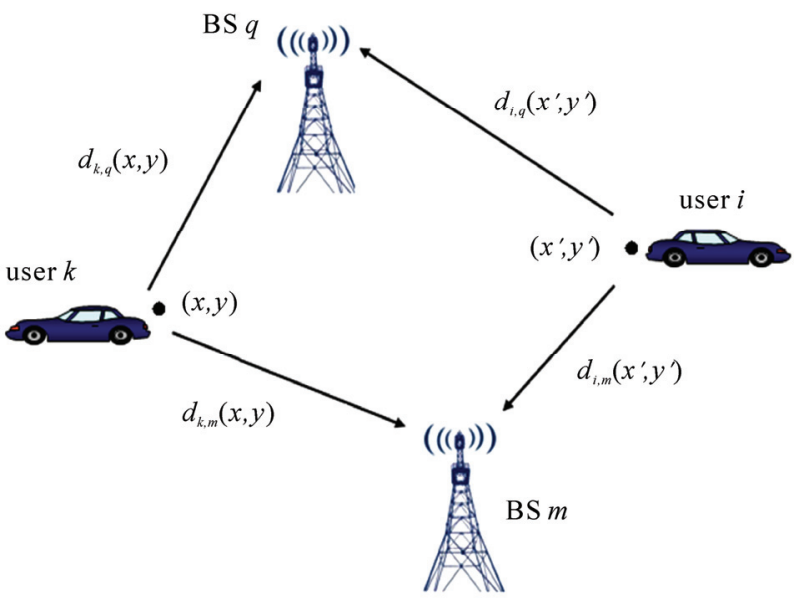

Figure 2. The distance between two pairs of mobile transmitters and base station receivers [12].

presence of closed-loop power control where $p_{k, m}$ is the transmitted power of user $k, m$ that in the case of the PPC, $p_{k, m}^{\prime}$ is fixed for all users within cell $m$ ( $p_{k, m}^{\prime}=E_{b} / T_{b}$ where $E_{b}$ is the energy per bit for all users) $[2,9]$.

The received signal in the base station $q$ in sensor $s$ for user $i, q$ is given by [9]

$$
\begin{aligned}
& r_{i, q, s}^{\prime}(t)=\sum_{l=1}^{L} \sqrt{p_{i, q}^{\prime}} b_{i, q}\left(t-\tau_{i, q, l}\right) c_{i, q}\left(t-\tau_{i, q, l}\right) \alpha_{i, q, l} \\
& \quad \times \exp \left(-j s k_{d} \sin \theta_{i, q, l}\right)+I_{i, q, s}(t)+n(t)
\end{aligned}
$$

where $I_{i, q, s}(t)$ is the interference for user $i, q$ in sensor $s$ and can be shown to be

$$
\begin{gathered}
I_{i, q, s}(t)=\sum_{m=1}^{M} \sum_{\substack{k=1 \\
k, m \neq i, q}}^{K_{m}} \sum_{l=1}^{L} \sqrt{p_{k, m}^{\prime} \Gamma_{k}(x, y)} b_{k, m}\left(t-\tau_{k, m, l}\right) \\
\times c_{k, m}\left(t-\tau_{k, m, l}\right) \alpha_{k, m, l} \exp \left(-j s k_{d} \sin \theta_{k, m, l}\right)
\end{gathered}
$$

where $K_{m}$ is the number of users in cell $m$ and $M$ is the number of base stations/cells.

\section{RAKE Receiver Performance Analysis}

The RAKE receiver structure in the DS-CDMA system is shown in Figure 1. The received signal is spatially processed by a beamforming circuit with CLMS algorithm, one for each resolvable path ( $L$ beamformers). The resultant signal is then passed on to a set of parallel matched filters (MFs), on a finger-by-finger basis. Also, the output signals from the $L$ matched filters are combined according to the conventional MRC principle and then are fed into the decision circuit of the desired user [9]. 


\subsection{Constrained LMS Algorithm}

It is well known that an array of $N$ weights has $N-1$ degree of freedom for adaptive beamforming $[9,16]$. This means that with an array of $N$ weights, one can generates $N-1$ pattern nulls and a beam maximum in desired directions. From (5), it is clear that the number of users is $K_{u}=\sum_{m=1}^{M} K_{m}$ and the number of interferes is $L K_{u}-1$. To null all of these interferes; one would have to have $L K_{u}$ weights, which is not practical. So, we focus only on the $L$ paths of the desired user. Thus, the minimum number of the antenna array weights is $L$ where, typically, $L$ varies from 2 to 6 [9].

In this paper, we use the CLMS adaptive beamforming algorithm. This algorithm is a gradient based algorithm to minimize the total processor output power, based on the look direction constraint. The adaptive algorithm is designed to adapt efficiently in agreement with the environment and able to permanently preserve the desired frequency response in the look direction while minimizing the output power of the array. The combined form of the constraint is called constraint for narrowband beamforming [12,17].

This form consider a narrowband beamformer where the narrowband signal from each element of smart antenna are multiplied by the complex weight calculated by using narrowband adaptive beamforming algorithm, and then summed to produce the output of the array. The definition of the complex weights of this beamformer in the $n$th iteration for user $i, q$ in the $j$ th path is as follows $[16,18]$.

$$
\mathbf{w}_{i, q}^{(j)}(n)=\left[w_{i, q, 0}^{(j)}(n) w_{i, q, 1}^{(j)}(n) \ldots w_{i, q, N-1}^{(j)}(n)\right]^{T}
$$

Accordingly, the output of the array in the $n$th iteration in the $j$ th path for user $i, q$ is given by

$$
y_{i, q}^{(j)}(n)=\mathbf{w}_{i, q}^{(j)}(n)^{H} \mathbf{r}_{i, q}^{\prime}(n)
$$

where $\quad \mathbf{r}_{i, q}^{\prime}=\left[\begin{array}{llll}r_{i, q, 0}^{\prime} & r_{i, q, 1}^{\prime} & \ldots & r_{i, q, N-1}^{\prime}\end{array}\right]^{T}$.

The expected output power of the array in the $n$th iteration is given by

$$
\begin{aligned}
\mathrm{E}\left(\mid y_{i, q}^{(j)}\right. & \left.\left.(n)\right|^{2}\right)=\mathrm{E}\left(y_{i, q}^{(j)}(n) y_{i, q}^{(j)}(n)^{*}\right) \\
& =\mathrm{E}\left(\mathbf{w}_{i, q}^{(j)}(n)^{H} \mathbf{r}_{i, q}^{\prime}(n) \mathbf{r}_{i, q}^{\prime}(n)^{H} \mathbf{w}_{i, q}^{(j)}(n)\right) \\
& =\mathbf{w}_{i, q}^{(j)}(n)^{H} \mathbf{R}_{r^{\prime} r^{\prime}} \mathbf{w}_{i, q}^{(j)}(n)
\end{aligned}
$$

where $\mathrm{E}($.$) is denoted the expectation and \mathbf{R}_{r^{\prime} r^{\prime}}$ is the correlation matrix of the received vector $\mathbf{r}_{i, q}^{\prime}(n)$.

A real-time CLMS algorithm for determining the optimal weight vector for user $i, q$ in the $j$ th path is
$[17,18]$ :

$$
\left\{\begin{array}{l}
\mathbf{w}_{i, q}^{(j)}(n+1)=\mathbf{w}_{i, q}^{(j)}(n)+\mu g\left(\mathbf{w}_{i, q}^{(j)}(n)\right) \\
\mathbf{w}_{i, q}^{(j) H} \mathbf{a}_{i, q}^{(j)}\left(\theta_{i, q, j}\right)=1
\end{array}\right.
$$

where

$$
\begin{aligned}
& \mathbf{a}_{i, q}^{(j)}\left(\theta_{i, q, j}\right)=\left[\begin{array}{ll}
1 & \exp \left(-j k_{d} \sin \theta_{i, q, j}\right) \ldots
\end{array}\right. \\
& \left.\ldots \exp \left(-j k_{d}(N-1) \sin \theta_{i, q, j}\right)\right]^{T}
\end{aligned}
$$

denotes spatial response of the array for user $i, q$ in the $j$ th path. Also in (9), $\mathbf{w}_{i, q}^{(j)}(n+1)$ is the new weight computed at the $(n+1)$ th iteration for user $i, q$ in the $j$ th path. Also, the variable scalar $\mu$ denotes a positive scalar (gradient step size) that controls the convergence characteristic of the algorithm, that is, how fast and how close the estimated weights approach the optimal weights, and $g\left(\mathbf{w}_{i, q}^{(j)}(n)\right)$ denotes an unbiased estimate of the gradient of the power surface $\left(\mathbf{w}_{i, q}^{(j)}(n)^{H} \mathbf{R}_{r^{\prime} r^{\prime}} \mathbf{w}_{i, q}^{(j)}(n)\right)$ which is the expected output power of the array) with respect to $\mathbf{w}_{i, q}^{(j)}(n)$ after the $n$th iteration. The algorithm is "constrained" because the weight vector satisfies the constraint at each iteration, that is $\mathbf{w}_{i, q}^{(j) H} \mathbf{a}_{i, q}^{(j)}\left(\theta_{i, q, j}\right)=1$. Rewrite the CLMS algorithm as follows [17].

$\mathbf{w}_{i, q}^{(j)}(n+1)=\boldsymbol{\beta}_{i, q}^{(j)}\left(\mathbf{w}_{i, q}^{(j)}(n)+\mu g\left(\mathbf{w}_{i, q}^{(j)}(n)\right)\right)+\frac{\mathbf{a}_{i, q}^{(j)}\left(\theta_{i, q, j}\right)}{N}$

where

$$
\boldsymbol{\beta}_{i, q}^{(j)}=\mathbf{I}-\frac{\mathbf{a}_{i, q}^{(j)}\left(\theta_{i, q, j}\right) \mathbf{a}_{i, q}^{(j)}\left(\theta_{i, q, j}\right)^{H}}{N}
$$

The gradient of $\mathbf{w}_{i, q}^{(j)}(n)^{H} \mathbf{R}_{r^{\prime} r^{\prime}} \mathbf{w}_{i, q}^{(j)}(n)$ with respect to $\mathbf{w}_{i, q}^{(j)}(n)$ is given by [17]

$$
\begin{aligned}
g\left(\mathbf{w}_{i, q}^{(j)}(n)\right) & \stackrel{\Delta}{=}-\frac{\partial}{\partial \mathbf{w}_{i, q}^{(j)^{*}}}\left(\mathbf{w}_{i, q}^{(j)}(n)^{H} \mathbf{R}_{r^{\prime} r^{\prime}} \mathbf{w}_{i, q}^{(j)}(n)\right) \\
& =-2 \mathbf{R}_{r^{\prime} r^{\prime}} \mathbf{w}_{i, q}^{(j)}(n)
\end{aligned}
$$

and its computation using this expression requires knowledge of $\mathbf{R}_{r^{\prime} r^{\prime}}$, which normally is not available in practice. For a standard LMS algorithm, an estimate of the gradient at each iteration is made by replacing $\mathbf{R}_{r^{\prime} r^{\prime}}$ by its noise sample $\mathbf{r}_{i, q}^{\prime}(n+1) \mathbf{r}_{i, q}^{\prime}(n+1)^{H}$ available at time instant $(n+1)$, leading to

$$
g\left(\mathbf{w}_{i, q}^{(j)}(n)\right)=-2 \mathbf{r}_{i, q}^{\prime}(n+1) y_{i, q}^{(j)^{*}}(n)
$$


The CLMS is a fast convergence algorithm. However, it is drastically sensitive to the mismatch in the direction of arrival. Meanwhile, the weights estimated by the standard algorithm are sensitive to the signal power, requireing a lower step size in the presence of a strong signal for the algorithm to converge, which in turn regarding the decrease of mis-adjustment error, the convergence time is increased $[17,19]$.

It should be mentioned that for the antenna arrays weight vector in the CLMS algorithm and for big $\mu$, will converge after a few iteration (is approximately equal to the number of beamformer weights, i.e., $n=N$ ) [19].

Accordingly, the output signal from the $j$ th beamformer $(j=1, \ldots, L)$ can be written as [9]

$$
\begin{aligned}
y_{i, q}^{(j)}(t) & =\sqrt{p_{i, q}^{\prime}} b_{i, q}\left(t-\tau_{i, q, j}\right) c_{i, q}\left(t-\tau_{i, q, j}\right) \alpha_{i, q, j} \\
& +\tilde{I}_{i, q}^{(j)}(t)+I_{i, q}^{(j)}(t)+n^{(j)}(t)
\end{aligned}
$$

where $n^{(j)}(t)$ is a zero mean Gaussian noise of variance $\sigma_{n}^{2}$ and $\tilde{I}_{i, q}^{(j)}(t)$, the IPI, is defined as

$$
\begin{aligned}
\tilde{I}_{i, q}^{(j)}(t) & =\sum_{\substack{l=1 \\
l \neq j}}^{L} \sqrt{p_{i, q}^{\prime}} g_{i, q}^{(j)}\left(\theta_{i, q, l}\right) \alpha_{i, q, l} b_{i, q}\left(t-\tau_{i, q, l}\right) \\
& \times c_{i, q}\left(t-\tau_{i, q, l}\right)
\end{aligned}
$$

and $I_{i, q}^{(j)}(t)$, the MAI, is defined as

$$
\begin{aligned}
I_{i, q}^{(j)}(t) & =\sum_{m=1}^{M} \sum_{\substack{k=1 \\
k, m \neq i, q}}^{K_{m}} \sum_{l=1}^{L} \sqrt{p_{k, m}^{\prime} \Gamma_{k}(x, y)} g_{i, q}^{(j)}\left(\theta_{k, m, l}\right) \\
\times & \times \alpha_{k, m, l} b_{k, m}\left(t-\tau_{k, m, l}\right) c_{k, m}\left(t-\tau_{k, m, l}\right)
\end{aligned}
$$

where $g_{i, q}^{(j)}(\theta)$ is the magnitude response of the $j$ th beamformer for user $i, q$ toward the DoA $\theta$ [9].

\subsection{Matched Filter}

Using beamforming in the first stage, will reduce the IPI for the desired user and the MAI from the other users whose signals arrive at different angles from the desired user signal (out-beam interference). Now, in the second stage of the RAKE receiver, the output signal from the $j$ th beamformer is directly passes on to a filter matched to the desired user's signature sequence. The $j$ th matched filter output corresponding to the $n$th bit is [9]:

$$
\begin{aligned}
z_{i, q}^{(j)}(n) & =\sqrt{p_{i, q}^{\prime}} b_{i, q}(n) \alpha_{i, q, j}+\tilde{I}_{i, q}^{\prime(j)}(n) \\
& +I_{i, q}^{\prime(j)}(n)+n^{\prime(j)}(n)
\end{aligned}
$$

where

$$
\begin{aligned}
& \tilde{I}_{i, q}^{\prime(j)}(n)=\frac{1}{T_{b}} \int_{(n-1) T_{b}+\tau_{i, q, j}}^{n T_{b}+\tau_{i, q, j}} \tilde{I}_{i, q}^{(j)}(t) c_{i, q}\left(t-\tau_{i, q, j}\right) d t \\
& I_{i, q}^{\prime(j)}(n)=\frac{1}{T_{b}} \int_{(n-1) T_{b}+\tau_{i, q, j}}^{n T_{b}+\tau_{i, q, j}} I_{i, q}^{(j)}(t) c_{i, q}\left(t-\tau_{i, q, j}\right) d t
\end{aligned}
$$

and

$$
n^{\prime(j)}(n)=\frac{1}{T_{b}} \int_{(n-1) T_{b}+\tau_{i, q, j}}^{n T_{b}+\tau_{i, q, j}} n^{(j)}(t) c_{i, q}\left(t-\tau_{i, q, j}\right) d t
$$

If we assume that the paths' delays from all users are less than the symbol duration $\left(\tau_{k, m, l}<T_{b}\right)$ for all users' signals on all paths, the $n$th bit IPI and MAI at the output of the $j$ th matched filter are expressed as [9]

$$
\begin{aligned}
\tilde{I}_{i, q}^{\prime(j)}(n) & =\sum_{\substack{l=1 \\
l \neq j}}^{L} \sqrt{p_{i, q}^{\prime}} g_{i, q}^{(j)}\left(\theta_{i, q, l}\right) \alpha_{i, q, l} b_{i, q}(n) \\
& \times R_{i, i}\left(\tau_{i, q, j}-\tau_{i, q, l}\right)
\end{aligned}
$$

and

$$
\begin{aligned}
I_{i, q}^{\prime(j)}(n) & =\sum_{m=1}^{M} \sum_{\substack{k=1 \\
k, m \neq i, q}}^{K_{m}} \sum_{l=1}^{L} \sqrt{p_{k, m}^{\prime} \Gamma_{k}(x, y)} g_{i, q}^{(j)}\left(\theta_{k, m, l}\right) \\
& \times \alpha_{k, m, l} b_{k, m}(n) R_{i, k}\left(\tau_{i, q, j}-\tau_{k, m, l}\right)
\end{aligned}
$$

where the autocorrelation function $R_{i, k}(\tau)$ is $[9,20]$ :

$$
R_{i, k}(\tau)=\frac{1}{T_{b}} \int_{T_{b}} c_{i, q}(t) c_{k, m}(t+\tau) d t
$$

If all users' delays are multiples of the chip period $\left(T_{c}\right)$, then

$$
R_{i, k}(\tau)=\frac{1}{G} \sum_{l_{1}=0}^{G-1} \sum_{l_{2}=0}^{G-1} c_{i, q}\left(l_{1}\right) c_{k, m}\left(l_{2}\right) R_{c}\left(\tau-\left(l_{1}-l_{2}\right) T_{c}\right)
$$

where the autocorrelation function $R_{c}(\tau)$ is:

$$
R_{c}(\tau)=\frac{1}{T_{b}} \int_{T_{b}} c(t) c(t+\tau) d t
$$

In the case of a maximal-length sequence (m-sequence) and for $0 \leq \tau \leq T_{b}$, we have [20]:

$$
R_{c}(\tau)= \begin{cases}1-\frac{|\tau|}{T_{c}}(1+1 / G) & ;|\tau| \leq T_{c} \\ -1 / G & ;|\tau| \geq T_{c}\end{cases}
$$

\subsection{Maximal Ratio Combining}

Diversity combining has been considered as an efficient way to combat multipath fading because the combined 
SINR is increased compared with the SINR of each diversity branch. The optimum combiner is the MRC whose SINR is the sum of the SINR's of each individual diversity branch [20,21].

After the finger-matched filter, the fingers' signals are combined according to the MRC principle in the third stage of the RAKE receiver. In this paper, we use the conventional MRC that the signal of user $i, q$ in the $j$ th path is combined using multiplying by the complex conjugate of $\alpha_{i, q, j}$.

The SINR in output of the RAKE receiver for user $i, q$ is $[9,21]$ :

$$
\operatorname{SINR}_{i, q}(\alpha)=\sum_{j=1}^{L} \operatorname{SINR}_{i, q}^{(j)}(\alpha)
$$

where

$$
\operatorname{SINR}_{i, q}^{(j)}(\alpha)=\frac{p_{i, q}^{\prime}\left|\alpha_{i, q, j}\right|^{2}}{\mathrm{E}\left(\tilde{I}_{i, q}^{\prime(j)}\right)^{2}+\mathrm{E}\left(I_{i, q}^{\prime(j)}\right)^{2}+\mathrm{E}\left(n^{\prime(j)}\right)^{2}}
$$

is the SINR in output of the RAKE receiver in path $j$ for user $i, q$.

Also, we can be rewritten the SINR in (29) by (30), that shown at the bottom of the page, where $\bar{\Gamma}_{k}(x, y)$

$$
=\mathrm{E}\left(\Gamma_{k}(x, y)\right) \text { and } \bar{\alpha}_{k, m, j}^{2}=\mathrm{E}\left(\left|\alpha_{k, m, j}\right|^{2}\right)[9,22] \text {. }
$$

In order to perform the BER, we assume Gaussian approximation for the probability density function of interference plus noise. The conditional BER for a BPSK modulation is $[9,20]$ :

$$
\operatorname{BER}_{i, q}(\alpha)=Q\left(\sqrt{2 \times \operatorname{SINR}_{i, q}(\alpha)}\right)
$$

where

$$
Q(x)=\frac{1}{\sqrt{2 \pi}} \int_{x}^{\infty} \exp \left(-u^{2} / 2\right) d u
$$

\section{Smart Step Closed-Loop Power Control Algorithm}

A major limiting factor for the satisfactory performance of CDMA systems is the near-far effect. Power control is an intelligent way of adjusting the transmitted powers in cellular systems so that the total transmit power (TTP) is minimized, but at the same time, the user SINRs satisfies the system quality of service (QoS) requirements [23].

Depending on the location where the decision on how to adjust the transmitted powers is made, the power control algorithm can be divided into two groups: centralized power control and distributed power control [1-6, 12]. In centralized power control, a network center can simultaneously compute the optimal power levels for all users. However, it requires measurement of all the link gains and the communication overhead between a network center and base stations. Thus, it is difficult to realize in a large system [24]. Distributed power control, on the other hand, uses only local information to determine transmitter power levels. It is much more scalable than centralized power control. However, transmitter power levels may not be optimal, resulting in performance degradation [25].

The distributed closed-loop power control problem has been investigated by many researchers from many perspectives during recent years $[4,23,26]$. For instance, the conventional fast closed-loop power control strategy used in practice in CDMA systems is a fixed step controller based on SINR measurements. The fixed step closed-loop power control (FSPC) algorithm is defined by [4]

$$
p_{i, q}^{n^{\prime}+1}=p_{i, q}^{n^{\prime}}+\delta \operatorname{sign}\left(\gamma_{i, q}^{*}-\gamma_{i, q}^{n^{\prime}}\right)
$$

where $p_{i, q}^{n^{\prime}}, \gamma_{i, q}^{*}$, and $\gamma_{i, q}^{n^{\prime}}$ are the transmitter power, SINR target, and measured SINR of user $i, q$ at time $n^{\prime}$, respectively, and $\delta$ is the fixed step size. Also $p_{i, q}^{n^{\prime}+1}$ is transmitter power control (TPC) command in the feedback link of the base station to user $i, q$ at time $n^{\prime}+1$ (all signals are in decibels).

Also, the distributed traditional closed-loop power control (DTPC) is defined by [23] the variance of

$$
p_{i, q}^{n^{\prime}+1}=\frac{\gamma_{i, q}^{*}}{\gamma_{i, q}^{n^{\prime}}} p_{i, q}^{n^{\prime}}
$$

In both algorithms, the simple intuition behind this iteration is that if the current SINR $\gamma_{i, q}^{n^{\prime}}$ of user $i, q$ is less than the target SINR $\gamma_{i, q}^{*}$, then the power of that user is increased; otherwise, it is decreased. It should be mentioned that convergence speed of DTPC algorithm is higher than FSPC algorithm. Also, the SINR mis-

$$
\frac{\operatorname{SINR}_{i, q}^{(j)}(\alpha)=}{p_{i, q}^{\prime} \bar{\alpha}_{i, q, j}^{2} \sum_{\substack{l=1 \\ l \neq j}}^{L}\left|g_{i, q}^{(j)}\left(\theta_{i, q, l}\right)\right|^{2} R_{i, i}^{2}\left(\tau_{i, q, j}-\tau_{i, q, l}\right)+\sum_{m=1}^{M} \sum_{\substack{k=1 \\ k, m \neq i, q}}^{K_{m}} p_{k, m}^{\prime} \bar{\Gamma}_{k}(x, y) \bar{\alpha}_{k, m, j}^{2} \sum_{l=1}^{L}\left|g_{i, q}^{(j)}\left(\theta_{k, m, l}\right)\right|^{2} R_{i, k}^{2}\left(\tau_{i, q, j}-\tau_{k, m, l}\right)+\frac{N_{0}}{2 T_{b}}}
$$


adjustment in FSPC algorithm is higher than DTPC algorithm. But, it has been shown that the FSPC algorithm converge to a bound region $\left|\gamma_{i, q}^{*}-\gamma_{i, q}^{n^{\prime}}\right| \leq 2 \delta k_{l d}$, where $k_{l d}$ is the loop delay [4].

Also in [26], variable step closed-loop power control (VSPC) algorithm has been proposed. In this algorithm, variable step size is discrete with mode $q_{v}$. It is shown that the performance of VSPC algorithm with mode $q_{v}=4$ is found to be worse than that of a fixed step algorithm $\left(q_{v}=1\right)$ under practical situations with loop delay of two power control intervals, but the convergence speed of VSPC algorithm is higher than FSPC algorithm. Also in this algorithm, the variance of the SINR mis-adjustment is reduced in compared to FSPC algorithm.

Practical implementations of power control in CDMA systems utilize closed-loop control, where the transmitter adjusts its power based on commands received from the receiver in a feedback channel. To minimize signaling overhead, typically one bit is used for the power control command. In practice, the command must be derived based on measurements made at the receiver, transmitted over the feedback channel to the transmitter, and finally processed and applied at the transmitter. All these operations constitute a loop delay, which can cause problems if it is not properly taken care of in the design of the power control algorithm. In many cases the loop delay is known due to a specific frame structure inherent in the system. A typical loop delay situation encountered in WCDMA systems is shown in Figure 3. The slot at time $n^{\prime} t$ is transmitted using power $p^{n^{\prime}}$. The receiver measures the SINR $\gamma^{n^{\prime}}$ over a number of pilot and/or data symbols and derives a TPC command. The command is transmitted to the transmitter in the feedback link and the transmitter adjusts its power at time $\left(n^{\prime}+1\right) t$ according to the command. It should be mentioned that since the power control signaling is standardized, the loop delays are known exactly [4].

In this paper, we propose the smart step closed-loop power control algorithm. The SSPC algorithm defines as follows.

$$
p_{i, q}^{n^{\prime}+1}=p_{i, q}^{n^{\prime}}+\delta\left|\gamma_{i, q}^{*}-\gamma_{i, q}^{n^{\prime}}\right| \operatorname{sign}\left(\gamma_{i, q}^{*}-\gamma_{i, q}^{n^{\prime}}\right)
$$

The SSPC algorithm is implemented as follows.

1) Select the initial transmitted power vector $\left(n^{\prime}=0\right)$ for all users within cell $m$ as

$$
\mathbf{p}_{m}^{0}=\left[\begin{array}{lll}
p_{1, m}^{0} & p_{2, m}^{0} \ldots p_{K_{m}, m}^{0}
\end{array}\right], \quad m=1,2, \ldots, M .
$$

2) Estimate the weight vector for all users with the CLMS algorithm using (11).

3) Calculate the SINR for all users using (28).

4) If $\left|\gamma_{k, m}^{*}-\gamma_{k, m}^{n^{\prime}}\right|>\varepsilon_{0}$ for each user then set $n^{\prime}=n^{\prime}+1$ and calculate the TPC for all users at time $n^{\prime}+1$ using (35) and go back to 2), where $\varepsilon_{0}$ is threshold value.

Finally, if $\left|\gamma_{k, m}^{*}-\gamma_{k, m}^{n^{\prime}}\right|<\varepsilon_{0}$ for all users then algorithm ends.

As will be seen from simulation results, because of variable coefficient in the sign function, the convergence speed of our algorithm is higher than VSPC and FSPC algorithms.

\section{BSA-MTP Technique}

The system capacity might be improved, if the users are

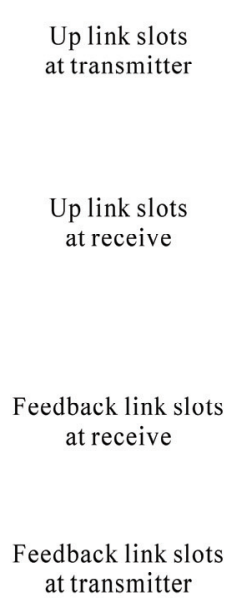

at transmitter

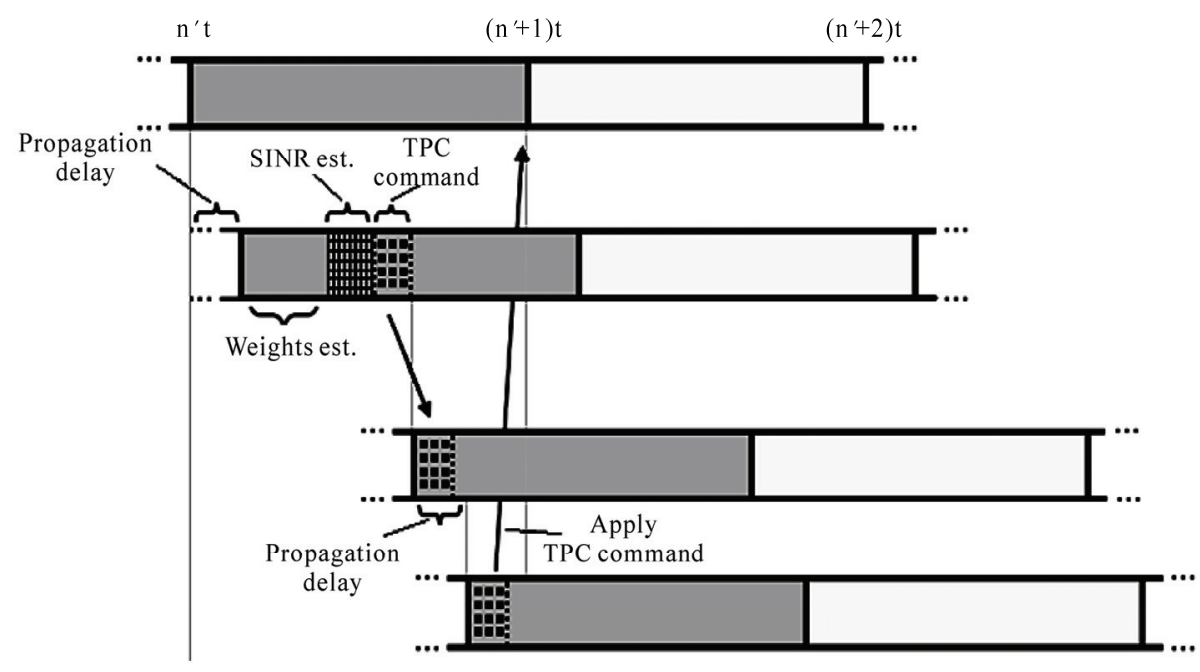

Figure 3. Example of power control timing in WCDMA systems [4]. 
allowed to switch to alternative base stations, especially when there are congested areas in the network. Obviously, when uplink performance is of concern, the switching should happen based on the total interferences seen by the base stations [15].

So far, we have considered the power control problem for a number of transmitter-receiver pairs with fixed assignments, which can be used in uplink or downlink in mobile communication systems. In an uplink scenario where base stations are equipped with antenna arrays, the problem of joint power control and beamforming, as well as base station assignment, naturally arises [12].

In this paper, we modify the BSA-MTP technique to support base station assignment as well. The modified technique can be summarized as follows.

1) Initially by the conventional BSA technique, each mobile connects to its base station, according to (2).

2) Estimate the weight vector for all users with the CLMS algorithm.

3) Each mobile updates its transmitted power based on the SSPC algorithm using (35).

4) Finally, $K_{r}=\left\lfloor K_{u} / M\right\rfloor$ users that their transmitted power is higher than the other users to be transferred to other base stations according to the following equation, where the function $\lfloor x\rfloor$ returns the integer portion of a number $x$.

$$
\Gamma_{k}(x, y)=\left\{\begin{array}{cl}
1 & ; k \in S_{\mathrm{BS} q} \\
\frac{\min _{m \in \Theta_{k}}\left\{d_{k, m}^{L_{\alpha}}(x, y) 10^{\xi_{k, m} / 10}\right\}}{d_{k, q}^{L_{\alpha}}(x, y) 10^{\xi_{k, q} / 10}} & ; k \in S_{\overline{\mathrm{BS} q}} \\
\frac{\min _{m \in \Theta_{k}}\left\{d_{k, m}^{L_{\alpha}}(x, y) 10^{\xi_{k, m} / 10}\right\}}{d_{k, q}^{L_{\alpha}}(x, y) 10^{\xi_{k, q} / 10}} & ; k \in S_{o}
\end{array}\right.
$$

where $S_{\overline{\mathrm{BS} q}}$ is the set of users that are in cell $q$ but not connected to $\operatorname{BS} q$ [2].

It should be mentioned that the technique for users that are present in the border of cells, the BER can be effecttively reduced.

\section{Switched-Beam Technique and Equal Sectoring Method}

One simple alternative to the fully adaptive antenna is the switched-beam architecture in which the best beam is chosen from a number of fixed steered beams. Switchedbeam systems are technologically the simplest and can be implemented by using a number of fixed, independent, or directional antennas [27]. We list the SB technique conditions for this paper as follows.
1) Coverage angle for all beams is $30^{\circ}$ and overlap between consecutive beams is $20^{\circ}$. Thus each base station has 36 beams.

2) Each user can be use one beam for its each path to communicate with a base station at any time

Also, one of simple methods to sectorize a cell is equal sectoring, in which all sectors have the same coverage angle. In this paper, we assume three sectors for each base station with sector angle $120^{\circ}$ for the ES method.

\section{Simulation Results}

We consider $M=4$ base stations for a four-cell CDMA system on a $2 \times 2$ grid as Figure 4. We assume a uniform linear array of $S$ omni-directional antennas in each base station with antenna spacing $d=\lambda / 2$. Also, we assume BPSK m-sequence code spreading with processing gain $G=64$; resolution $R=1$; the input data rate $T_{b}=9.6 \mathrm{kbps}$; the number of antenna weights $N=3$; the number of antenna sensors $S=3$; threshold value $\varepsilon_{0}=0.1 \mathrm{~dB}$; frequency-selective fading channel with $L=2$ resolvable propagation paths; variance of the complex Gaussian fading channel coefficient $\sigma_{\alpha}^{2}$ $=4 \mathrm{~dB}$; fixed step size for SSPC, FSPC, and VSPC algorithms $\delta=0.01$; mode $q_{v}=4$ for VSPC algorithm [26]; variance of the log-normal shadow fading $\sigma_{\xi}^{2}=8 \mathrm{~dB}$; path-loss component $L_{\alpha}=4$; initial value for weight vectors in the CLMS algorithm $\mathbf{w}(0)=\mathbf{0}$; initial value for transmitted power vectors $\mathbf{p}_{m}^{0}=\mathbf{0}$. The SINR target value is the same for all users and is set to $\gamma^{*}=5(7 \mathrm{~dB})$. It also is assumed that the distribution of users in all cells is uniform.

First, in order to compare the BSA-MTP and conventional BSA techniques, we assume the PPC, and the BER

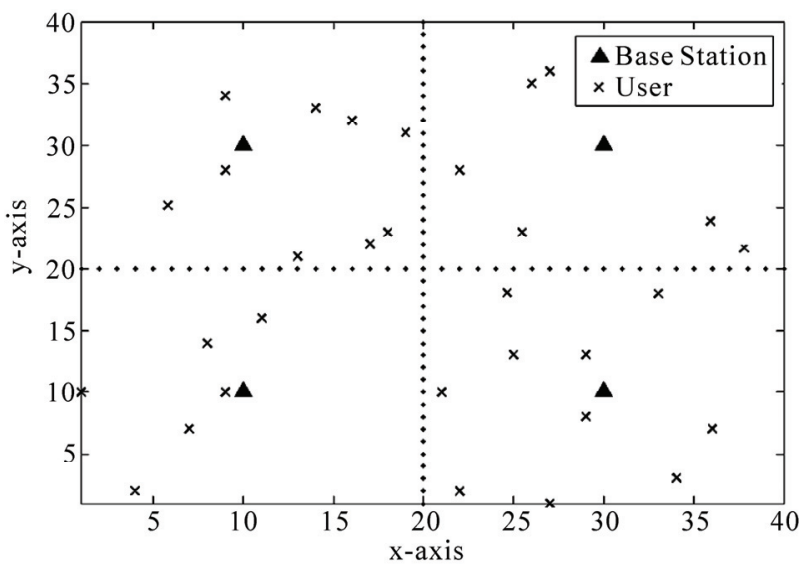

Figure 4. Location plot of base stations and users in four cells. 
has been calculated from (31). Finally, we compare the TTP with the joint SSPC algorithm and BSA-MTP technique in comparison with other methods.

Figure 5 shows the average BER versus the signal-to-noise ratio (SNR) for different receivers (one, two, and three-stage receivers) in the case of $K_{u}=32$ active users and the PPC case. It should be mentioned that in this simulation, $K_{r}=8$ users can to be transferred to other base stations with the BSA-MTP technique. Also in this simulation we use CLMS algorithm or SB technique in the first stage of the RAKE receiver. It is clear that, in MF only receiver (one-stage receiver) and in the case of the conventional BSA technique, we still have the error floor at high SNR. Using CLMS and MRC receiver (two-stage RAKE receiver) or CLMS, $\mathrm{MF}$, and MRC receiver (the three-stage RAKE receiver as Figure 1) has a better performance than using MF only. Also observe that using the BAS-MTP technique in the case of three-stage RAKE receiver (CLMS method in the first stage), the average BER is lower than the conventional BSA technique. For example, at a SNR of $20 \mathrm{~dB}$, the average BER is 0.0096 for the three-stage RAKE receiver with the conventional BSA technique, while for the BSA-MTP technique, the average BER is 0.0031 . Also it can be seen that the average BER in the SB technique is less than the CLMS algorithm. Also, it is clear that the MAI is not removed totally and the performance is still worse than the single user per cell bound.

Figure 6 shows the average BER versus the number of active users $\left(K_{u}\right)$ for different receivers as Figure 5, in the case of the PPC and SNR $=10 \mathrm{~dB}$. At a BER of 0.005 , the three-stage RAKE receiver (CLMS method in the first stage) with the BSA-MTP technique support $K_{u}=29$ users, while for the three-stage RAKE receiver and the conventional BSA technique support $K_{u}=18$ users. We also observe that the three-stage RAKE receiver can achieve lower BER than the one and twostage receivers. Also at a BER of 0.002 , the three-stage RAKE receiver for the SB technique in the first stage and for the BSA-MTP technique support $K_{u}=49$ users, while the CLMS, MF, and MRC receiver support $K_{u}=18$ users. It should be mentioned that increasing the number of active users in the SB technique, will lead more complexity in receiver in comparison with the CLMS algorithm. Also increasing the number of active users $\left(K_{u}\right)$, will increase the number of users that can to be transferred to other base stations $\left(K_{r}\right)$ in the BSAMTP technique.

Figure 7 shows the comparison of the average SINR achieved over $K_{u}=32$ users versus the power control iteration index $\left(n^{\prime}\right)$ for SSPC, VSPC, and FSPC algorithms and for BSA-MTP and conventional BSA tech

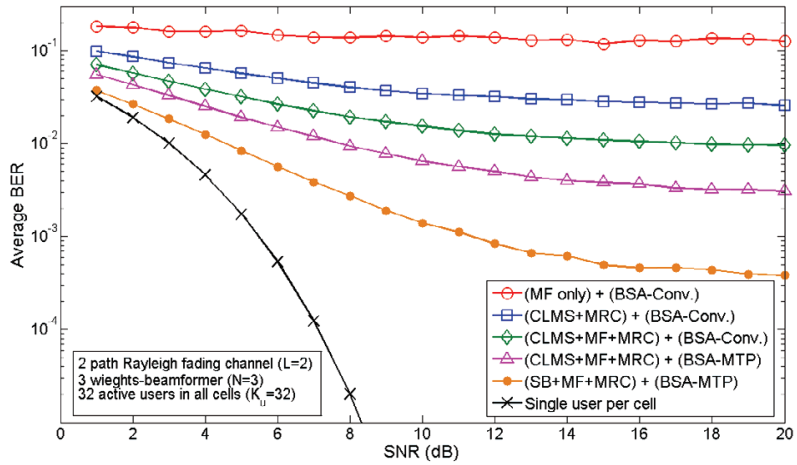

Figure 5. Average BER of all users versus the SNR for the PPC case.

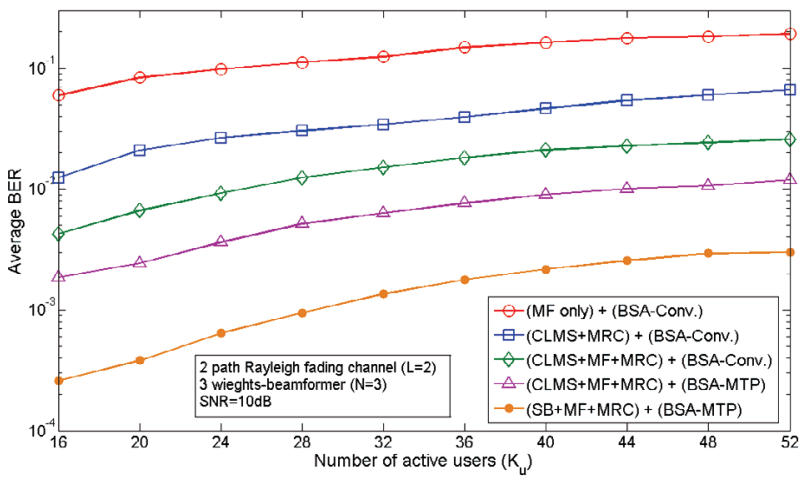

Figure 6. Average BER for all users versus the number of active users for the PPC case and $S N R=10 \mathrm{~dB}$.

niques. In this simulation, the three-stage RAKE receiver uses CLMS, SB, or ES methods in the first stage. Also, we assume that each user to have a maximum power constraint of 1 watt. Accordingly, we observe that the convergence speed of the SSPC algorithm is faster than the VSPC and FSPC algorithms. The figure also shows that the SSPC algorithm with the BSA-MTP technique converges faster than the SSPC algorithm for the conventional BSA technique. In addition, we see that the convergence speed of the SSPC algorithm for the SB technique is faster than the CLMS and ES methods. Also observe that the average SINR level achieved is below the target SINR value for the ES method, because in this method, the MAI is much higher than SB technique and CLMS algorithm.

Figure 8 shows the comparison of TTP usage versus the power control iteration index $\left(n^{\prime}\right)$ when there are $K_{u}=32$ users in all cells according to Figure 7. But in this simulation, we assume that users have no maximum power constraints. Similar to Figure 7, we observe that the ES method never can achieve the target SINR value. Also this figure shows that the SSPC algorithm offers more savings in the TTP as compared to the VSPC and FSPC algorithms. In addition, the figure shows that the 


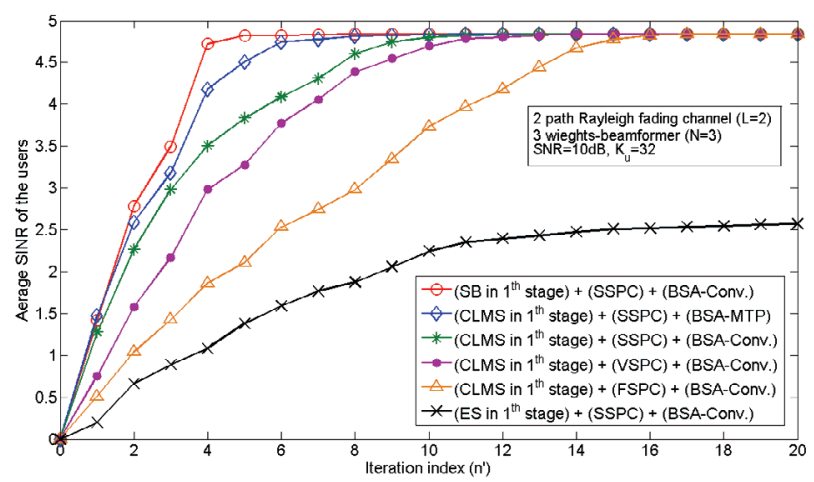

Figure 7. Average SINR of all users versus power control iteration index with maximum power constraint of 1 watt.

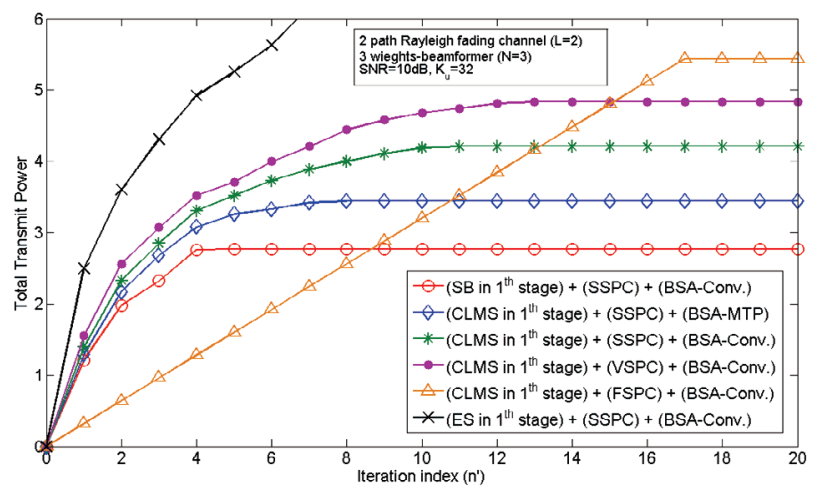

Figure 8. Total transmit power of all users versus power control iteration index. No power constraints.

TTP in BSA-MTP technique is less than conventional BSA technique. Also it can be seen that the TTP for the SB technique is lower than the CLMS algorithm, because in SB technique, the MAI is lower than CLMS algorithm.

\section{Conclusions}

In this paper, we studied the RAKE receiver performance of multiple-cell DS-CDMA system with the space diversity processing, Rayleigh frequency-selective channel model, closed-loop power control, and base station assignment. This receiver consists of CLMS, MF, and MRC in three stages.

Accordingly, we proposed the SSPC algorithm and the BSA-MTP technique to reduce the CCI and the MAI. It has been shown that, by using antenna arrays at the base stations, the SSPC algorithm and the BSA-MTP technique will decrease the interference in all cells. In addition, it can be seen that the TTP in the SSPC algorithm is less than the VSPC and FSPC algorithms. Also our results show that the TTP for BSA-MTP technique is lower than conventional case. Thus, it decreases the BER by allowing the SINR targets for the users to be higher, or by increasing the number of users supportable at a fixed SINR target level. On the other hand, it has been shown that the convergence speed of the SSPC algorithm is increased in comparison with the VSPC and FSPC algorithms. It has also observed that using the BSA-MTP technique will decrease the average BER of the system to support a significantly larger number of users.

\section{References}

[1] A. Abrardo and D. Sennati, "On the Analytical Evaluation of Closed-Loop Power-Control Error Statistics in DS-CDMA Cellular Systems," IEEE Transactions on Vehicular Technology, Vol. 49, No. 6, 2000, pp. 20712080.

[2] L. Carrasco and G. Femenias, "Reverse Link Performance of a DS-CDMA System with Both Fast and Slow Power Controlled Users," IEEE Transactions on Wireless Communications, Vol. 7, No. 4, 2008, pp. 1255-1263.

[3] L. Qian and Z. Gajic, "Variance Minimization Stochastic Power Control in CDMA System," IEEE Transactions on Wireless Communications, Vol. 5, No. 1, 2006, pp. 193202.

[4] M. Rintamaki, H. Koivo and I. Hartimo, "Adaptive ClosedLoop Power Control Algorithms for CDMA Cellular Communication Systems," IEEE Transactions on Vehicular Technology, Vol. 53, No. 6, 2004, pp. 1756-1768.

[5] J. Wang and A. Yu, "Open-Loop Power Control Error in Cellular CDMA Overlay Systems," IEEE Journal on Selected Areas in Communications, Vol. 19, No. 7, 2001, pp. 1246-1254.

[6] J. T. Wang, "Admission Control with Distributed Joint Diversity and Power Control for Wireless Networks," IEEE Transactions on Vehicular Technology, Vol. 58, No. 1, 2009, pp. 409-419.

[7] M. Dosaranian-Moghadam, H. Bakhshi, G. Dadashzadeh and P. Rahmati, "Adaptive Beamforming Method Based on Constrained LMS Algorithm for Tracking Mobile User," IEEE Global Mobile Congress, Shanghai, October 2009, pp. 1-6.

[8] J. Chang, L. Tassiulas and F. Rashid-Farrokhi, "Joint Transmitter Receiver Diversity for Efficient Space Division Multiaccess," IEEE Transactions on Wireless Communications, Vol. 1, No. 1, 2002, pp. 16-27.

[9] N. A. Mohamed and J. G. Dunham, "A Low-Complexity Combined Antenna Array and Interference Cancellation DS-CDMA Receiver in Multipath Fading Channels," IEEE Journal on Selected Areas in Communications, Vol. 20, No. 2, 2002, pp. 248-256.

[10] N. A. Mohamed and J. G. Dunham, "Adaptive Beamforming for DS-CDMA Using Conjugate Gradient Algorithm in a Multipath Fading Channel," Proceedings of 1999 IEEE Emerging Technologies Symposium, Dallas, April 1999, pp. 859-863.

[11] F. Rashid-Farrokhi, K. J. Ray-Liu and L. Tassiulas, "Transmit Beamforming and Power Control for Cellular Systems," IEEE Journal on Selected Areas in Communications, Vol. 
16, No. 8, 1998, pp. 1437-1450.

[12] F. Rashid-Farrokhi, L. Tassiulas and K. J. Ray-Liu, "Joint Optimal Power Control and Beamforming in Wireless Networks Using Antenna Arrays," IEEE Transactions on Communications, Vol. 46, No. 10, 1998, pp. 1313-1324.

[13] R. D. Yates and C. Huang, "Integrated Power Control and Base Station Assignment," IEEE Transactions on Vehicular Technology, Vol. 44, No. 3, 1995, pp. 638-644.

[14] S. V. Hanly, "An Algorithm for Combined Cell-Site Selection and Power Control to Maximize Cellular Spread Spectrum Capacity," IEEE Journal on Selected Areas in Communications, Vol. 13, No. 7, 1995, pp. 1332-1340.

[15] M. Mahmoudi and E. S. Sousa, "Joint Power Control, Base Station Assignment and Sectorization for CDMA Cellular Systems," Proceedings of 2000 IEEE Vehicular Technology Conference, Boston, September 2000, pp. 573-580.

[16] J. Litva and T. Kwok-Yeung, "Digital Beamforming in Wireless Communications," Artech House, Boston, 1996.

[17] X. Y. Sun, X. H. Lian and J. J. Zhou, "Robust Adaptive Beamforming Based on Maximum Likelihood Estimation," International Conference on Microwave and Millimeter Wave Technology, Nanjing, April 21-24, 2008, pp. 11371140.

[18] M. Z. Shakir and T. S. Durrani, "Narrowband Beamforming Algorithm for Smart Antennas," International Bhurban Conference on Applied Sciences \& Technology, Islamabad, Pakistan, January 2007, pp. 49-54.

[19] S. Haykin, "Adaptive Filter Theory," 3rd Edition, Prentice Hall, New Jersey, 1996.
[20] R. L. Peterson, R. E. Ziemer and D. E. Borth, "SpreadSpectrum Communications," Prentice-Hall, New Jersey, 1995.

[21] N. Kong and L. B. Milstein, "Average SNR of a Generallzed Diversity Selection Combining Scheme," IEEE Communications Letters, Vol. 3, No. 3, 1999, pp. 57-59.

[22] J. C. Liberti and T. S. Rappaport, "Smart Antennas for Wireless Communications IS-95 and Third Generation CDMA Applications," Prentice-Hall, New Jersey, 1999.

[23] A. Yener, R. D. Yates and S. Ulukus, "Interference Management for CDMA Systems through Power Control, Multiuser Detection, and Beamforming," IEEE Transactions on Communications, Vol. 49, No. 9, 2001, pp. 1227-1239.

[24] S. Grandhi, R. Vijayan and D. Goodman, "Centralized Power Control in Cellular Radio Systems," IEEE Transactions on Vehicular Technology, Vol. 42, No. 4, 1993, pp. 466-468.

[25] S. Grandhi, R. Vijayan and D. Goodman, "Distributed Power Control in Cellular Radio Systems," IEEE Transactions on Communications, Vol. 42, No. 2-4, 1994, pp. 226-228.

[26] A. Kurniawan, "Effect of Feedback Delay on Fixed Step and Variable Step Power Control Algorithm in CDMA Systems," The 8th International Conference on Communication Systems, Indonesia, 2002, pp. 1096-1100.

[27] B. Allen and M. Beach, "On the Analysis of Switchedbeam Antennas for the W-CDMA Downlink," IEEE Transactions on Vehicular Technology, Vol. 53, No. 3, 2004, pp. 569-578. 